\title{
Daytime running lights in the USA: what is the impact on vehicle crashes in Minnesota?
}

\author{
Michele E. Krajicek • Raquel M. Schears
}

Received: 28 April 2009 /Accepted: 29 November 2009/Published online: 26 March 2010

(C) Springer-Verlag London Ltd 2010

\begin{abstract}
Background Daytime running lights (DRLs) are a safety feature intended to reduce crashes by increasing the contrast between vehicles and the background.

Aims The purpose of this study was to determine whether there is an association between vehicles in the USA being equipped with DRLs and crash rates.

Methods This was a retrospective study using the Minnesota Department of Transportation (MNDOT) Crash Database from 1995 to 2002. Crash reports included in the analyses were limited to accidents involving vehicles 1995 or newer (DRLs not available on prior models) and limited to ideal conditions: (1) daylight, (2) optimal visibility, and (3) dry road surface. The vehicle identification number (VIN) was used to determine the make, model, and year. This information was cross-referenced with a National Highway Traffic Safety Administration table of manufacturer listed DRL conditions to determine vehicle DRL status. Crude crash rates for vehicles were calculated relative to the number of all registered vehicles in Minnesota in 2004, for models 1995-2002. Ninety-five percent confidence intervals (CI) for the rates were constructed assuming a Poisson error distribution.

Results During 1995-2002, there were 184,637 vehicles (1995 or newer) with identifiable VINs involved in accidents which occurred under the specified test con-
\end{abstract}

The views expressed in this paper are those of the author(s) and not those of the editors, editorial board or publisher.

\footnotetext{
M. E. Krajicek $\cdot$ R. M. Schears $(\square)$

Mayo Clinic College of Medicine,

Rochester, MN, USA

e-mail: Schears.Rocky@mayo.edu

M. E. Krajicek

e-mail: krajicek.michele@mayo.edu
}

ditions. Of these vehicles, 37,909 were determined to have standard DRLs and 146,728 were determined to be models without DRLs (including those listed as DRL optional). The crash rate among vehicles without standard DRLs was 1.73 (95\% CI: $1.71-1.75)$ times higher than the rate for vehicles with standard DRLs. The rate ratio was also significant for fatal vehicle crash rates 1.48 (95\% CI: 1.23 1.76).

Conclusion Minnesota vehicles equipped with DRLs were associated with a statistically significant lower crash rate compared to vehicles without DRLs from 1995 to 2002.

Keywords Daytime running lights · Crashes · Motor vehicle accidents $\cdot$ Headlights $\cdot$ Automobile

\section{Introduction}

Daytime running lights (DRLs) are a safety feature intended to reduce crashes by increasing the contrast between vehicles and the background. Currently, Finland, Sweden, Norway, Canada, Denmark, Hungary, and Iceland all require vehicle lights during daytime hours. Most of the studies of the effectiveness of DRLs have been done in Scandinavia. Finland was the first to institute DRL legislation in rural areas, and literature reports a $27 \%$ crash rate reduction [1]. In 1977, Sweden started requiring the use of daytime vehicle lights on all roads, and reduction of crash rates from 9 to $21 \%$ were reported by Andersson and Nilsson [2]. Norway began to require installation of DRLs in all new cars beginning in 1985 and use of daytime lights on all vehicles by 1988 . A $15 \%$ crash rate reduction for crashes involving more than one vehicle was later reported by Elvik [3]. Lastly, Denmark has required use of DRLs on all roads since 1990 , with a statistically significant $37 \%$ rate 
reduction for crashes involving a left turn in a study by Hansen [4]. A 1995 paper by Theeuwes and Riemersma criticized the odds ratio methodology of all these early studies [6]. In response, a meta-analysis of 17 studies by Elvik estimated a decrease in crash rate of $10-15 \%$ for multivehicle crashes and total crash reduction of 3-12\% [7].

The first studies of DRLs in North America were done on fleet vehicles. In a study by Stein, corporate fleet vehicles in the USA equipped with DRLs had 7\% fewer relevant crashes compared to the group of fleet vehicles without DRLs during 1983-1984 [8]. Sparks et al. reported $15 \%$ crash reduction in government fleet vehicles in Canada equipped with DRLs [9]. By December 1989 all newly manufactured vehicles in Canada were required to be equipped with DRLs, and within 4 years, Arora et al. reported a statistically significant $8 \%$ reduction in relevant collisions [10].

DRLs in non-fleet passenger vehicles have been introduced more recently in the USA. In 1995, Volvo and Saab were first to install DRLs on all their new cars sold in the USA. By 1997, all new Suzuki, Volkswagen, and General Motors models included DRLs. Yet a decade later, only a few studies and reports have been published regarding the use of daytime headlights in the USA. Farmer and Williams used a casecontrol method to analyze multiple vehicle daytime crashes in nine states for a group of vehicles equipped with DRLs. They reported that these vehicles were involved in $3.2 \%$ fewer crashes [11]. The National Highway Traffic Safety Administration (NHTSA) reported a preliminary assessment in June 2000. Using the Fatality Analysis Reporting System (FARS), they analyzed fatal crashes in four states from 1995 to 1997. They found no significant difference in risk of two vehicle opposite-direction crashes comparing vehicles with DRLs to vehicles without DRLs. However, using the State Data System (SDS) from Florida, Maryland, Missouri, and Pennsylvania, a statistically significant $7 \%$ reduction in risk for relevant (including crash subtypes presumably affected by DRLs, such as opposite-direction) nonfatal crashes was identified, and DRL-equipped vehicles were associated with $28 \%$ fewer pedestrian fatalities [12].

In this study, we tested the hypothesis that passenger vehicles in the USA equipped with DRLs are associated with decreased crash rates compared to those without DRLs under "high test" weather (daylight and optimal visibility) and road (dry) conditions.

\section{Methods}

This was a retrospective study using the Minnesota Department of Transportation (MNDOT) Crash Database from 1995 to 2002. Vehicle crashes, for which police reports were filed, were cross-verified and matched against the NHTSA archival registry maintained for research purposes. Definitions of "crash" and "fatality" were based on the terminology referenced by MNDOT Traffic Accident Report (form version: PS-32003-10) as documented by police authorities at the time of the actual accident. Specifically, fatalities recorded were for any scene deaths immediately related to the motor vehicle collision. Crash reports included in the analyses were limited to crashes involving automobiles, pickups, and vans and crashes that occurred under high test weather and road conditions all defined a priori. The high test conditions included: (1) temporal limitations to daylight, defined as dawn to dusk, (2) optimal visibility, defined as clear or cloudy, and (3) road surface identified as dry. Studied vehicles were also limited to models 1995 and newer, since prior models did not have DRLs. The vehicle identification number (VIN) of vehicles involved in crashes was used to determine the specific make, model, and year. This information was crossreferenced with a NHTSA table of manufacturer listed DRL conditions to determine each vehicle DRL status.

Crash rates for vehicles with standard DRL and without DRL feature were calculated as relative to the number of all registered vehicles in Minnesota with or without the DRL feature, respectively. The number of registered vehicles in Minnesota was determined from the MNDOT vehicle registration file obtained in 2004 for models 1995-2002. In 2004, the number of these vehicles, with and without standard DRLs, was 788,840 and $1,763,134$, respectively. MNDOT does not keep a retrospective database of registered vehicles. Therefore, the only total number of vehicles which can be obtained is a number in real time. This number was obtained in 2004, at the time the study was started. Use of this single-year denominator assumes that the proportion of vehicles with and without the standard DRL feature was constant over the years of this study. Although the rates will be overestimated since the denominators represent a single year, the rate ratios will be appropriate if the previous assumption holds. Ninety-five percent confidence intervals (CI) for the rates were constructed using a Poisson error distribution. The two rates were compared using a two-sided $\mathrm{F}$ test for the ratio of two Poisson random variants.

\section{Results}

During the 7-year study period, 184,637 vehicles (1995 or newer) had identifiable VINs and were involved in accidents that occurred under the specified test conditions. Of these vehicles, 37,909 were determined to have standard DRLs and 146,728 were determined to be models without DRLs (Fig. 1). The standard DRL group had a higher percentage of automobiles vs pickups and vans (78.5\%) 


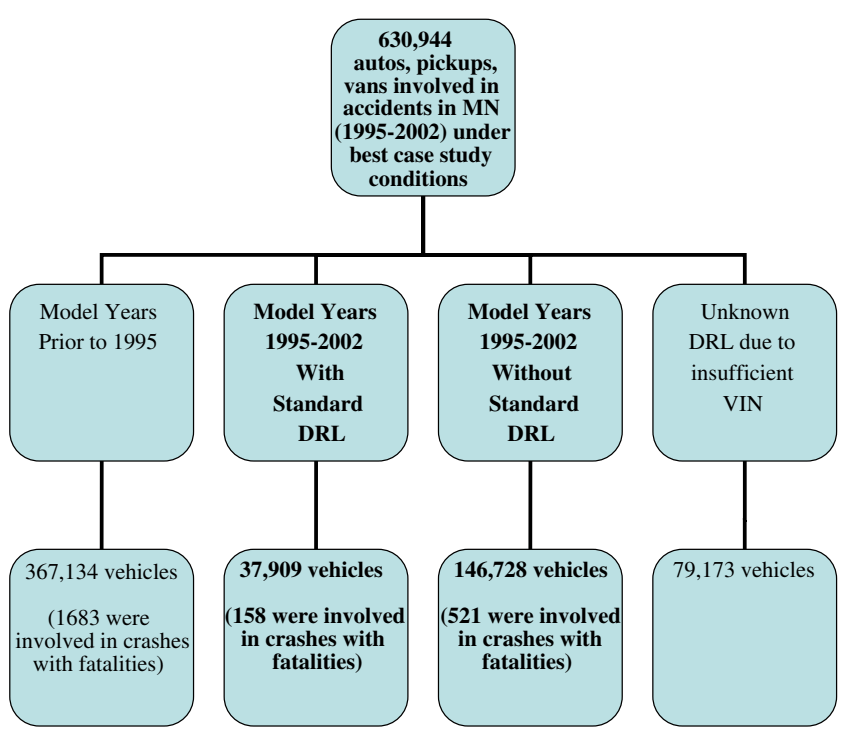

Fig. 1 Summary of vehicles identified for analysis

than the group without standard DRLs (66.3\%). Other accident characteristics were similar between the standard vs nonstandard DRL groups (Table 1).

The crash rate per 10,000 vehicles among vehicles with standard DRLs was $481(37,909 / 788,840$; 95\% CI: 476485). For vehicles without standard DRLs the crash rate per 10,000 was 832 (146,728/1,763,134; 95\% CI: 828-836). The rate ratio was 1.73 (95\% CI: $1.71-1.75 ; p<0.001)$ (Table 2).

Crashes were also analyzed based on whether a fatality was reported. The rate of fatal vehicle crashes for vehicles with standard DRLs in Minnesota between 1995 and 2002 was 2.0 per $10,000(158 / 788,840 ; 95 \%$ CI: $1.7-2.3)$. The rate of fatal vehicle crashes for vehicles without standard DRLs was 3.0 per $10,000(521 / 1,763,134$; $95 \%$ CI: $2.7-$ $3.2)$. The rate ratio was $1.48(95 \%$ CI: $1.23-1.76$; $p<$ 0.001) (Table 2).

Vehicle crashes were divided by the type of collision, including collisions with other vehicles, pedestrians, and bicycles. (Table 1). Of the 37,909 vehicles with standard DRLs involved in accidents, 34,475 were involved in collisions with other vehicles. This is a crash rate of 437 per 10,000 vehicles (95\% CI: 432-442). Of the 146,728 vehicles without standard DRLs involved in accidents, 133,892 were involved in collisions with other vehicles. This is a crash rate of 759 per 10,000 vehicles $(95 \%$ CI: 755-764). The rate ratio for vehicles involved in collisions with other vehicles was 1.74 (95\% CI: $1.72-1.76 ; p<0.001)$ (Table 2). A total of 230 vehicles with standard DRLs were involved in collisions with pedestrians, which is a crash rate of 2.9 per 10,000 vehicles (95\% CI: 2.5-3.3). In comparison, a total of 911 vehicles without standard DRL were involved in collisions with pedestrians, which is a crash rate of 5.2 per 10,000 vehicles (95\% CI: 4.8-5.5) (Table 2). The rate ratio for vehicles involved in collisions with pedestrians was 1.77 (95\% CI: $1.53-2.05 ; p<0.001)$. Finally, for collisions with a bicycle, there were 358 vehicles with standard DRLs involved in such collisions for a crash rate of 4.5 per 10,000 vehicles. Without standard DRLs, 1,379 vehicles were involved in collisions with bicycles for a crash rate of 7.8 per 10,000 vehicles. The rate ratio for vehicles involved in collisions with bicycles is $1.72(95 \%$ CI: $1.54-1.94 ; p<0.001$. (Table 2)

\section{Discussion}

Based on our study results, DRLs had an association with vehicle crash reduction in motor vehicle collisions, consistent with two previous studies. Farmer and Williams showed that vehicles equipped with DRLs were involved in $3.2 \%$ fewer crashes [11]. The NHTSA reported a $7 \%$ reduction in risk of relevant nonfatal crashes [12]. Our crude crash rate reduction as reflected by the rate ratio was notably higher than in both of these previous studies. This may be due to the fact that our study was a retrospective study of all vehicle crashes in Minnesota during the time period, whereas the preceding studies cited employed a case-control methodology to compare specific subsets of vehicles with and without DRLs.

Our study shows a statistically significant reduction in fatal crashes for vehicles with DRLs versus those without DRLs. The NHTSA report found no significant reduction in fatal crashes [5]. This latter finding may be attributable to the relatively low numbers of vehicles involved in fatal crashes compared to all crashes reflected in the NHTSA study denominator. Larger studies with greater numbers of fatal crashes would be helpful to further delineate the impact of DRLs in fatal crashes where causation is likely multifactorial.

Vehicles that collided with other vehicles showed lower crash rate in vehicles with standard DRLs compared to those without DRLs. This is a subtype of crashes that would expectedly be impacted by the DRL feature, as increased visibility of other vehicles would likely decrease collisions [13]. In addition, the rate of vehicles colliding with pedestrians may also be predictably lowered by the use of DRLs because these vehicles may be increasingly visible to pedestrians. Our study does demonstrate a reduction in vehicle-pedestrian crashes not inconsistent with the $28 \%$ reduction rate reported by the NHTSA [5]. To our knowledge, no traffic law revisions, such as lower speed limitations, or newer primary seat belt stop legislations, affected our crash rates. Specifically, there were no traffic law changes in Minnesota identified during the study period. 
Table 1 Vehicle characteristics by DRL status
${ }^{\mathrm{a}}$ Where percentages do not equal 100 , categories labeled "not applicable" and "other" were incomplete and therefore not included in the data set

\begin{tabular}{|c|c|c|}
\hline & $\begin{array}{l}\text { Std. DRL, year 1995+, } \\
N=37,909(\%)\end{array}$ & $\begin{array}{l}\text { No std. DRL, year } 1995+\text {, } \\
N=146,728(\%)\end{array}$ \\
\hline \multicolumn{3}{|l|}{ Vehicle type } \\
\hline Automobile & $29,750(78.5)$ & $97,317(66.3)$ \\
\hline Pickup & $5,600(14.8)$ & $30,959(21.1)$ \\
\hline Van & $2,559(6.8)$ & $18,452(12.6)$ \\
\hline \multicolumn{3}{|l|}{ Type of accident ${ }^{\mathrm{a}}$} \\
\hline Collision with vehicle & $34,475(90.9)$ & $133,892(91.3)$ \\
\hline Collision with train & $15(<0.1)$ & $30(<0.1)$ \\
\hline Collision with bike & $358(0.9)$ & $1,379(0.9)$ \\
\hline Collision with pedestrian & $230(0.6)$ & $911(0.6)$ \\
\hline \multicolumn{3}{|l|}{ Diagram $^{\mathrm{a}}$} \\
\hline Rear end & $13,721(36.2)$ & $52,700(35.9)$ \\
\hline Sideswipe passing & $2,396(6.3)$ & $9,379(6.4)$ \\
\hline Left turn into oncoming & $2,412(6.4)$ & $9,723(6.6)$ \\
\hline Ran off road, left side & $539(1.4)$ & $2,145(1.5)$ \\
\hline Right angle & $7,979(21)$ & $30,347(20.7)$ \\
\hline Right turn into cross traffic & $218(0.6)$ & $814(0.6)$ \\
\hline Ran off road, right side & $728(1.9)$ & $3,019(2.1)$ \\
\hline Head on & $499(1.3)$ & $2,147(1.5)$ \\
\hline Sideswipe opposing & $406(1.1)$ & $1,612(1.1)$ \\
\hline \multicolumn{3}{|l|}{ Road description $^{\mathrm{a}}$} \\
\hline Freeway (including ramps) & $5,701(15)$ & $21,698(14.8)$ \\
\hline Other divided highway & $6,054(16)$ & $22,640(15.4)$ \\
\hline One-way street & $793(2.1)$ & $3,409(2.3)$ \\
\hline 4-6 lane undivided, $2-3$ each & $7,063(18.6)$ & $27,380(18.7)$ \\
\hline 3 lanes undivided & $482(1.3)$ & $1,735(1.2)$ \\
\hline 2 lanes, 1 each way & $11,894(31.4)$ & $45,577(31.1)$ \\
\hline Alley, driveway & $149(0.4)$ & $603(0.4)$ \\
\hline Private property & $153(0.4)$ & $486(0.3)$ \\
\hline \multicolumn{3}{|l|}{ Functional class $^{\mathrm{a}}$} \\
\hline Rural & 6,717 (17.7) & $23,685(16.1)$ \\
\hline Urban & $30,152(79.5)$ & $118,558(80.8)$ \\
\hline
\end{tabular}

\section{Limitations}

Our study has at least four limitations. First, unknown DRL status excluded vehicles from analysis, and incremental value of layered standards or options in crash prevention is

Table 2 Crash rate ratios: vehicles with DRLs versus vehicles without DRLs

\begin{tabular}{lc}
\hline All vehicles & 1.74 \\
\hline Vehicles involved in fatal crashes & 1.48 \\
Vehicles involved: & \\
In collisions with other vehicles & 1.74 \\
In collisions with pedestrians & 1.77 \\
In collisions with bicycles & 1.72 \\
\hline
\end{tabular}

not quantified. Second, snapshots of data streams may fail to demonstrate the whole picture in complex large volume relationships over time. We used a denominator from vehicles registered in 2004 and assumed a similar proportion of vehicles with DRL standard to those without DRL standard for all of the study years. We believe the proportion of vehicles remained reasonably constant over the study period, but there is no retrospective database to confirm this. Third, use of "best-case scenario" assumptions to disprove the null hypothesis may limit capture of other significant differences between groups. Lastly, confounders related to the driver or vehicle parameters such as age, experience, or safety record may significantly affect associations. Driver and vehicle files containing private or privileged information (insurance status, license qualifications, organ donor information, health outcomes, etc.) were not accessible for the purpose of this research study. 


\section{Conclusion}

Minnesota vehicles equipped with DRLs were associated with a statistically significant decrease in crash rates compared to vehicles without DRLs, model year 1995 or newer, from 1995 to 2002. These improvements followed nonmandatory DRL implementation by select manufacturers in the USA.

\section{References}

1. Andersson K, Nilsson G, Salusjärvi M (1976) The effect on traffic accidents of the recommended use of vehicle running lights in the daytime in Finland (in Swedish). Report 102, National Road and Traffic Research Institute, Linköping

2. Andersson K, Nilsson G (1981) The effects on accidents of compulsory use of running lights during daylight in Sweden. Report 208A, National Road and Traffic Research Institute, Linköping

3. Elvik R (1993) The effects on accidents of compulsory use of daytime running lights for cars in Norway. Accid Anal Prev 25 (4):383-398

4. Hansen LK (1993) Daytime running lights in Denmarkevaluation of the safety effect. Danish Council of Road Safety Research, Copenhagen

5. Holló P (1998) Changes in the legislation on the use of daytime running lights by motor vehicles and their effect on road safety in Hungary. Accid Anal Prev 30(2):183-199

6. Theeuwes J, Riemersma J (1995) Daytime running lights as a vehicle collision countermeasure: the Swedish evidence reconsidered. Accid Anal Prev 27(5):633-642

7. Elvik R (1996) A meta-analysis of studies concerning the safety effects of daytime running lights on cars. Accid Anal Prev 28 (6):685-694
8. Stein H (1985) Fleet experience with daytime running lights in the United States. SAE Technical Paper 851239. Society of Automotive Engineers, Warrendale

9. Sparks GA, Neudorf RD, Smith AE et al (1993) The effect of daytime running lights on crashes between two vehicles in Saskatchewan: a study of a government fleet. Accid Anal Prev 25:619-625

10. Arora H, Collard D, Robbins G et al (1994) Effectiveness of daytime running lights in Canada. Report TP 12298 (E), Transport Canada, Ottawa

11. Farmer C, Williams A (2002) Effects of daytime running lights on multiple-vehicle daylight crashes in the United States. Accid Anal Prev 34:197-203

12. National Highway Traffic Safety Administration (2000) A preliminary assessment of the crash-reducing effectiveness of passenger car daytime running lamps (DRLs) DOT HS 808 645, June

13. Williams AF, Lancaster KA (1995) The prospects of daytime running lights for reducing vehicle crashes in the United States. Public Health Rep 110(3):233-239

Michele Krajicek is an Emergency Medicine Attending Physician at the Saint Marys Hospital, the Mayo Clinic tertiary referral center and Level I Trauma Center serving southeastern Minnesota. She is an Instructor in the Mayo College of Medicine with research interests in injury prevention and public health surveillance activities.

Raquel Schears is a practicing Emergency Medicine Attending Physician at the Saint Marys Hospital, the Mayo Clinic tertiary referral center and Level I Trauma Center serving southeastern Minnesota. She is an Assistant Professor at the Mayo Medical College with an academic focus in public health medical ethics. She teaches clinically and mentors scholarly projects as faculty and staff within the Department of Emergency Medicine and Residency Training Program. 